\title{
Revisiting Ginsberg and Rubinstein's study
}

Hiroshi Date, MD

\author{
From the Department of Thoracic Surgery, Graduate School of Medicine, Kyoto University, Kyoto, Japan. \\ Disclosures: Author has nothing to disclose with regard to commercial support. \\ Received for publication May 5, 2017; accepted for publication May 11, 2017; available ahead of print June 16, \\ 2017. \\ Address for reprints: Hiroshi Date, MD, Department of Thoracic Surgery, Graduate School of Medicine, \\ Kyoto University, 54 Kawahara-cho, Shogoin, Sakyo-ku, Kyoto 606-8507, Japan (E-mail: hdate@ kuhp. \\ kyoto-u.ac.jp). \\ J Thorac Cardiovasc Surg 2017;154:1109 \\ $0022-5223 / \$ 36.00$ \\ Copyright (c) 2017 by The American Association for Thoracic Surgery \\ http://dx.doi.org/10.1016/j.jtcvs.2017.05.030
}

Lobectomy with mediastinal dissection is currently the standard surgical procedure for clinical stage IA nonsmall-cell lung cancer. This is largely dependent on a single and ever completed prospective study comparing sublobar resection with lobectomy reported by Ginsberg and Rubinstein in $1995 .{ }^{1}$ A higher rate of locoregional recurrence was found after sublobar resection compared with after lobectomy. In the era of ground glass opacity, segmentectomy is being increasingly applied not only for high-risk patients, but also for low-risk healthy patients. Solid tumor size or ratio and maximum standardized uptake value (SUVmax) have been identified as independent predictors for pathological tumor aggressiveness. ${ }^{2-4}$ Therefore, they appear to be important parameters to consider when selecting patients for sublobar segmentectomy without compromising longterm survival. For properly selected patients, segmentectomy has been reported to be suitable for clinical stage IA lung adenocarcinoma, with survival rates equivalent to those for lobectomy. However, previous meta-analyses on this topic have found somewhat contradictory results. Taioli and colleagues ${ }^{5}$ reported that among 20 studies reporting recurrence rate, 11 favored lobectomy and 9 favored sublobar resection, because these studies were heterogeneous in design and analysis.

In this issue of the Journal, Hattori and colleagues ${ }^{6}$ analyze clinicopathological data of 200 surgically resected clinical stage IA lung adenocarcinomas. Lobectomy was performed in the majority of patients $(\mathrm{n}=165 ; 82.5 \%)$, with sublobar resection chosen in selected patients $(\mathrm{n}=35 ; 17.5 \%)$. This study is unique in that it included only radiologically pure solid tumors; thus, the subjects were relatively homogeneous. The 3-year locoregional recurrence-free survival of the sublobar resection arm was significantly worse than that of the lobectomy arm when the tumor had an SUVmax $>3.3(62.7 \%$ vs $82.9 \%$; $P=.0281$ ). The authors concluded that lobectomy should be the choice for patients with pure solid adenocarcinoma with high SUVmax.

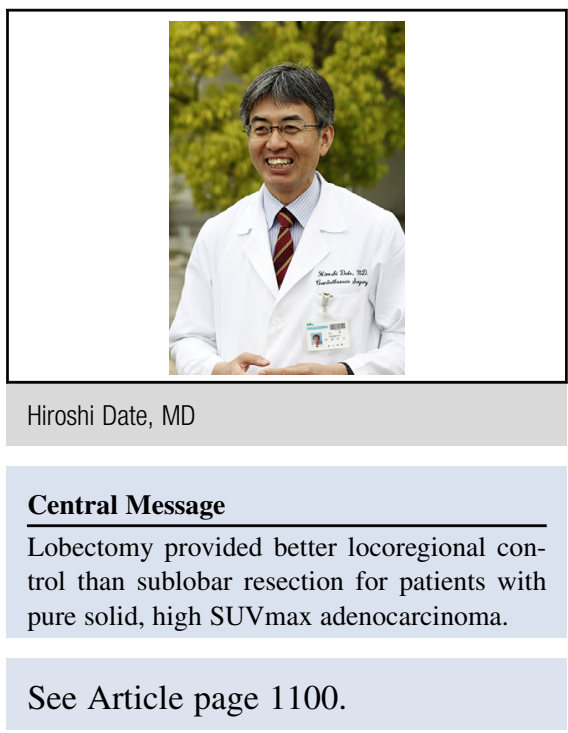

In the prospective study of Ginsberg and Rubinstein, 247 patients were randomized between 1982 and 1988. Patients were eligible without computed tomography evaluation, which was not widely available in those days. Of course, positron emission tomography scans were not available in routine practice either. Most of the tumors were detected by plain chest roentgenogram; therefore, we can assume that they were radiologically pure solid tumors, similar to the subjects analyzed in the study of Hattori and colleagues. It is interesting that both studies reached the same conclusion, that lobectomy provided better locoregional control than sublobar resection.

\section{References}

1. Ginsberg RH, Rubinstein LV. Randomized trial of lobectomy versus limited resection for T1N0 non-small cell lung cancer. Lung Cancer Study Group. Ann Thorac Surg. 1995;60:615-23.

2. Suzuki K, Kusumoto M, Watanabe S, Tsuchiya R, Asamura H. Radiologic classification of small adenocarcinoma of the lung: radiologic-pathologic correlation and its prognostic impact. Ann Thorac Surg. 2006;81:413-9.

3. Tsutani Y, Miyata Y, Nakayama H, Okumura S, Adachi S, Yoshimura M, et al Prediction of pathologic node-negative clinical stage IA lung adenocarcinoma for optimal candidates undergoing sublobar resection. J Thorac Cardiovasc Surg. 2012;144:1365-71.

4. Tsutani Y, Miyata Y, Nakayama H, Okumura S, Adachi S, Yoshimura M, et al. Oncologic outcomes of segmentectomy compared with lobectomy for clinical stage IA lung adenocarcinoma: propensity score-matched analysis in a multicenter study. J Thorac Cardiovasc Surg. 2013;146:358-64.

5. Taioli ET, Yip R, Olkin I, Wolf A, Nicastri D, Henschke C, et al. Survival after sublobar resection for early-stage lung cancer: methodological obstacles in comparing the efficacy to lobectomy. J Thorac Oncol. 2015;11:400-6.

6. Hattori A, Matsunaga T, Takamochi K, Oh S, Suzuki K. Indications for sublobar resection of clinical stage IA radiologic pure-solid lung adenocarcinoma. J Thorac Cardiovasc Surg. 2017; 154:1100-8. 